\title{
Exploiting Media Stream Similarity for Energy-Efficient Decoding and Resource Prediction
}

JUAN HAMERS and LIEVEN EECKHOUT, Ghent University, Belgium

This article introduces a novel approach to energy-efficient media stream decoding that is based on the notion of media stream similarity. The key idea is that platform-independent scenarios with similar decoding complexity can be identified within and across media streams. A device that decodes a media stream annotated with scenario information can then adjust its processor clock frequency and voltage level based on these scenarios for lower energy consumption. Our evaluation, done using the H.264 AVC decoder and 12 reference video streams, shows an average energy reduction of $44 \%$ while missing less than $0.2 \%$ of the frame deadlines using scenario-driven video decoding.

An additional application of scenario-based media stream annotation is to predict required resources (compute power and energy) for consuming a given service on a given device. Resource prediction is extremely useful in a client-server setup in which the client requests a media service from the server or content provider. The content provider (in cooperation with the client) can then determine what service quality to deliver, given the client's available resources. Scenario-aware resource prediction can predict (compute power and energy) consumption with errors less than $4 \%$ (and an overall average $1.4 \%$ error).

Categories and Subject Descriptors: C.3 [Special-Purpose and Application-Based Systems]: Real-time and embedded systems

General Terms: Design, Performance, Experimentation

Additional Key Words and Phrases: Video decoding, Video stream similarity, Scenario-based design, Energyefficiency, Resource prediction

\section{ACM Reference Format:}

Hamers, J. and Eeckhout, L. 2012. Exploiting media stream similarity for energy-efficient decoding and resource prediction. ACM Trans. Embedd. Comput. Syst. 11, 1, Article 2 (March 2012), 25 pages.

DOI $=10.1145 / 2146417.2146419$ http://doi.acm.org/10.1145/2146417.2146419

\section{INTRODUCTION}

Energy consumption is a key design issue for many of today's systems. This is especially the case for battery-operated devices such as laptops, handheld computers, mobile phones, PDAs, digital cameras, audio/video players, etc. Multimedia applications, and video applications in particular, are increasingly popular applications on most of these devices.

This article proposes a novel approach to media decoding that substantially reduces energy consumption. Our approach is based on the notion of media stream similarities,

This paper combines and extends two previously published conference papers, namely, Hamers and Eeckhout [2007] and Hamers et al. [2007].

J. Hamers was supported by a BOF grant from Ghent University. This research was also partly supported by the FWO projects G.0160.02 and G.0255.08.

Authors' addresses: J. Hamers and L. Eeckhout, ELIS Department, Ghent University, SintPietersnieuwstraat 41, B-9000 Gent, Belgium; email: \{jmhamers, leeckhou\}@elis.UGent.be.

Permission to make digital or hard copies of part or all of this work for personal or classroom use is granted without fee provided that copies are not made or distributed for profit or commercial advantage and that copies show this notice on the first page or initial screen of a display along with the full citation. Copyrights for components of this work owned by others than ACM must be honored. Abstracting with credit is permitted. To copy otherwise, to republish, to post on servers, to redistribute to lists, or to use any component of this work in other works requires prior specific permission and/or a fee. Permissions may be requested from Publications Dept., ACM, Inc., 2 Penn Plaza, Suite 701, New York, NY 10121-0701 USA, fax +1 (212) 869-0481, or permissions@acm.org.

(C) 2012 ACM 1539-9087/2012/03-ART2 $\$ 10.00$

DOI 10.1145/2146417.2146419 http://doi.acm.org/10.1145/2146417.2146419 
both within and across streams. An offline platform-independent analysis, which is to be done at the content provider side, determines media stream intervals (An interval is a single frame or a sequence of frames) with similar complexity across the various media streams in the database. These similarly complex intervals are determined based on their encoding and are called scenarios. The key point is that all the intervals from a given scenario exhibit a similar decoding complexity, that is, require similar compute power and energy consumption at decode time. Scenario information is annotated to the media streams in the content provider's database. The client then needs to be profiled using scenario representatives in order to associate platform-specific tuning to the platform-independent scenarios. The client then uses the scenario information encoded in the media stream for driving the energy-efficient decoding: a low complexity interval can be decoded at a lower frequency and voltage, thereby reducing energy consumption, while meeting the soft real-time deadlines.

An important feature of scenario-annotated media streams is that it also allows for predicting the required compute power and energy resources for decoding a given input stream. This is referred to as resource prediction. In particular, based on the per-scenario client profiling, the content provider can determine whether the client is powerful enough for meeting the deadlines for most of the frames and/or whether the available battery lifetime will be sufficient for decoding the entire media stream. The client or the content provider can then decide whether to download or send the media stream, or download or send the same media content in a different format that the client can handle, given the available resources. In other words, resource prediction allows for the content provider to determine what service quality to deliver.

Scenario-aware decoding and resource prediction are applicable to a wide variety of media decoders. In many multimedia system environments there is a content provider that provides video streams, audio streams, etc., as a service to its end users. The client that runs the decoder is very often battery-operated and resource-constrained. Resource prediction and resource-aware media decoding are thus very important issues at the client side. The media stream decoding application that we specifically focus on in this article is video decoding; however, our scheme is also applicable to other media stream-processing applications, such as audio stream decoding, (e.g., MP3 decoding).

This paper makes the following contributions.

-We introduce and exploit the notion of intra/intermedia stream similarity. To the best of our knowledge, there is no prior work that describes and exploits media stream similarity. More specifically towards video decoding, we identify video stream similarity based on macroblock information of the encoded video stream. Using 12 video streams and detailed cycle-level processor simulation, we show, that the platformindependent intra- and inter video stream scenarios correlate very well with decoding complexity at the client side.

-We show that video stream similarity has an important application for energyefficient video decoding. The platform-independent scenario information can be translated into platform-dependent decode information, in terms of client-side optimal processor clock frequency and voltage level. By doing so, the client can decode a video stream at reduced energy consumption while meeting soft real-time deadlines. Our experimental results using the H.264 AVC video decoder show that our approach achieves a $44 \%$ reduction in energy consumption while missing less than $0.2 \%$ of the frame deadlines, on average.

- Scenario-based media stream annotation enables resource prediction, or the prediction of required energy and compute power resources for decoding a media stream. We apply scenario-aware resource prediction for predicting decode time, decode 




Fig. 1. Annotating video streams with scenario information by the content provider.

energy, and quality of service. Our experimental results show good accuracy for all three purposes. For example, our method predicts decode time and energy with a worst-case error of less of than $4 \%$ and an average $1.4 \%$ error.

This article is organized as follows. We first discuss how scenarios are to be identified and how media streams are to be annotated with scenario information at the content provider side. We subsequently discuss how these scenarios can be used to drive energy-efficient client-side decoding and resource prediction. After explaining our experimental setup, we then evaluate the energy-efficiency of scenario-based media decoding and the accuracy of scenario-based resource prediction. And finally, after discussing related work, we conclude.

\section{SCENARIO IDENTIFICATION BY THE CONTENT PROVIDER}

Figure 1 illustrates how video streams are annotated with scenario information by the content provider. The content provider maintains a database of video streams; these video streams are shown in the left top corner of Figure 1. The content provider collects a macroblock profile for all of the video streams in the database. A macroblock profile counts the number of macroblocks of a given type per video stream interval. We define a video stream interval as a frame or a sequence of subsequent frames. The interval granularities that we consider in this article are a single frame and a group-of-pictures (GOP), which is a sequence of 16 subsequent frames. The purpose of a macroblock profile is to characterize the decoding complexity in a platform-independent way, that is, a macroblock profile is independent of the decoder as well as the system on which the video stream is to be decoded.

Once a macroblock profile is collected for all the video streams in the database, all the intervals can be viewed as points in a multidimensional space, which we call the interval space (see Figure 1). The various dimensions in the interval space are the macroblock types, that is, each dimension represents one macroblock type. The interval space is 22-dimensional in our setup. We then apply cluster analysis in the interval space. Cluster analysis finds groups of intervals, which we call scenarios, based on their macroblock characteristics. The idea is that the intervals of a given scenario show similar macroblock characteristics, whereas intervals from different scenarios show dissimilar macroblock characteristics. Note that cluster analysis is 
done on the collection of intervals from all video streams in the database. As such, scenarios consisting of intervals from different video streams may be formed. In other words, we identify platform-independent similarities at the interval level within a given video stream (intra-video stream similarity), as well as across various video streams (inter-video stream similarity).

Once the scenarios are identified, we then annotate all the video streams in the content provider's database with scenario information. The annotation is done at the interval level by adding scenario identifiers. In other words, if the interval granularity is a single frame, the content provider provides scenario information per individual frame; if the granularity is a GOP, the content provider provides scenario information per GOP.

Note that identifying the scenarios in the interval space is a one-time cost, that is, profiling an initial set of video streams, as well as identifying the scenarios in the interval space, needs to be done only once. Whenever a new video stream is added to the database, a macroblock profile needs to be computed for the new video stream, and the video stream intervals need to be annotated with scenario information. In other words, our framework does not require that the cluster analysis be redone, nor that all the video streams in the database be reannotated whenever a new video stream is added to the database. However, whenever appropriate, the content provider may want to update the database with new scenario information.

As a follow-on to the preceding discussion, it is important to note that scenario annotation can be done in both an offline as well as an online setup. Offline scenario annotation is done when all video streams reside on a storage device, and need to be processed to compute scenario IDs. This is a typical use case for a server: the content provider providing a service to its end users. Online scenario annotation, on the other hand, is done on-the-fly while encoding a video stream, for example, by a video recording device. Once the characteristics for the various scenarios are known, the video encoder can simply classify frames or GOPs on-the-fly, according to their macroblock profile.

We now discuss in more detail the following issues related to scenario identification by the content provider: (i) macroblock profiling, (ii) identifying video stream scenarios using cluster analysis, and (iii) annotating the video stream with scenario information.

\subsection{Macroblock Profiling}

Macroblock profiling captures a platform-independent signature of the coding complexity at the interval level. Although the following discussion on macroblock profiling is geared toward the H.264/AVC decoder [Ostermann et al. 2004] that we target in this work, similar profiles can be computed for other types of media streams. A macroblock consists of $16 \times 16$ picture elements. We identify the following macroblock types. There are 21 macroblock types in total.

-An intra-prediction macroblock only uses already transmitted macroblocks of the same frame for predicting samples of the given macroblock. There are two flavors of intra-prediction macroblocks, namely $16 \times 16$ (type 1 ) and $4 \times 4$ (type 2 ). The $4 \times 4$ macroblock consists of $164 \times 4$ subblocks, which are separately encoded.

-An inter-prediction, or a motion-compensated macroblock, uses previously transmitted frames for predicting samples of the given macroblock. An inter prediction macroblock can be divided into four partitions, namely $16 \times 16$ (type 3 ), $16 \times 8$ (type 4 ), $8 \times 16$ (type 5 ) and $8 \times 8$. The $8 \times 8$ submacroblock can be further subdivided into $8 \times 4,4 \times 8$, and $4 \times 4$ partitions. As such, we consider 15 macroblock, types for $8 \times 8$ macroblocks, depending on the partitioning within the submacroblocks.

- The final macroblock type is the skip macroblock type (type 21).

For each of these 21 macroblocks, macroblock profiling computes the number of each of these macroblock types per interval. Note that not all of the above macroblock 
types appear for all frame types, for example, I frames do not contain inter-prediction macroblocks. In such a case, the macroblock type count for that frame equals zero.

Next to these macroblock types, macroblock profiling also measures the residual byte count per interval. (The residual bytes allow the decoder to correct the predictions based on the macroblock encoding.) In order to bring the residual byte count in the range of the macroblock profiles (note that we will use the macroblock profile to build the interval space), we first divide the residual byte count with its standard deviation, and subsequently multiply it with the total number of macroblocks in the interval.

\subsection{Video Stream Scenario Identification}

We identify video stream scenarios through cluster analysis in the interval space. Cluster analysis [Johnson and Wichern 2002] is a data analysis technique that is aimed at clustering $n$ cases (in our case, video stream intervals) based on the measurements of $p$ variables (in our case, macroblock characteristics). The final goal is to obtain a number of groups containing intervals that exhibit similar behavior. There exist two commonly used types of clustering techniques, namely linkage clustering and K-means clustering. We now discuss these two clustering techniques.

Linkage clustering starts with a matrix of distances between the $n$ cases or intervals. As a starting point for the algorithm, each interval is considered a group. In each iteration of the algorithm, the two groups that are most close to each other (with the smallest linkage distance) will be combined to form a new group. As such, close groups are gradually merged until, finally, all cases are in a single group. This can be represented in a so-called dendrogram, which graphically represents the linkage distance for each group merge at each iteration of the algorithm. Having obtained a dendrogram, it is up to the user to decide how many clusters to take. This decision can be made based on the linkage distance: small linkage distances imply strong clustering, while large linkage distances imply weak clustering. The distance measure that we use in the interval space is the Manhattan distance. There exist several methods for calculating the distance between groups or clusters, potentially leading to different clustering results. In this article, we consider the furthest neighbor strategy, also known as complete linkage. In complete linkage, the distance between two clusters is computed as the largest distance between any two members from the respective clusters.

Another approach to cluster analysis is $K$-means clustering. K-means clustering produces exactly $K$ clusters with the greatest possible distinction. The algorithm works as follows. In each iteration, the distance is calculated for each case to the center of each cluster. A case is then assigned to the closest cluster, and new cluster centers are computed. This algorithm is iterated until no more changes are observed. It is well known that the result of K-means clustering is dependent on the choice of the initial cluster centers.

Linkage clustering has the advantage that the clustering needs to be done only once and that various cluster sizes can be explored based on this single clustering. K-means clustering, on the other hand, has the advantage of being computationally more efficient than linkage clustering, especially when a large number of cases need to be clustered. As such, K-means clustering may be the method of choice for large video stream databases. In our work, we use both linkage clustering and K-means clustering; however, because of space constraints, we only present results for linkage clustering. The results for K-means clustering are very similar.

\subsection{Video Stream Scenario Annotation}

Annotating the video streams with scenario information can be done in basically two ways. One possibility is that the scenario information is embedded in the video stream, that is, the scenario identifier (ID) for a given interval is added to the interval header. 


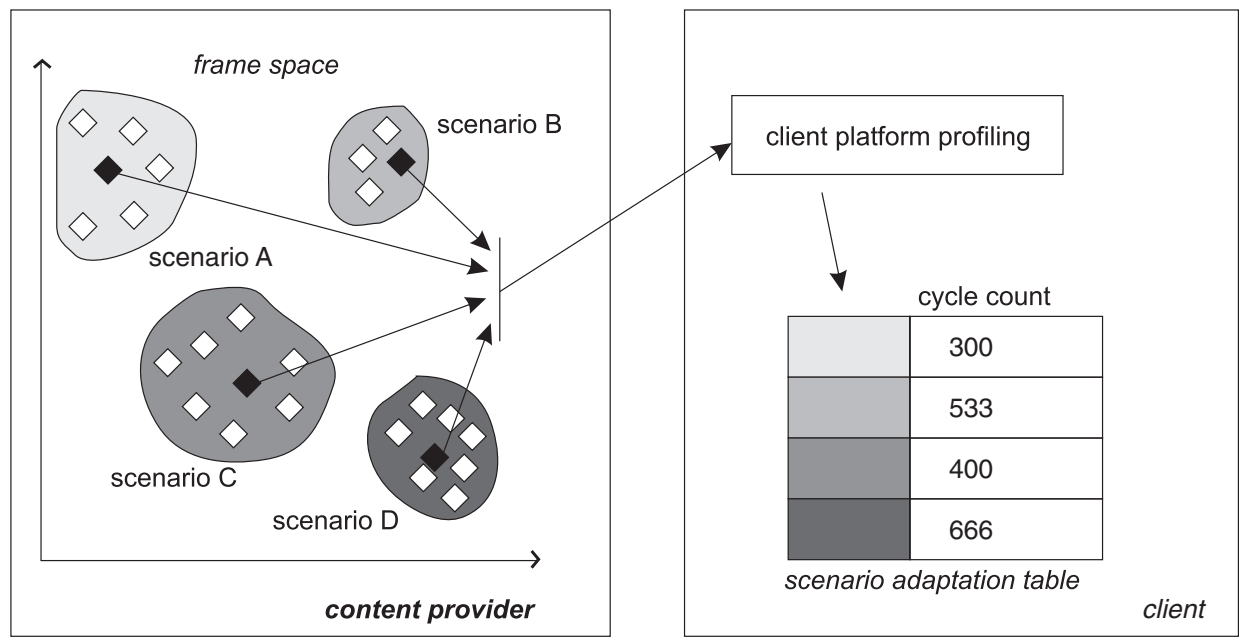

Fig. 2. Filling the scenario adaptation table at the client side.

This may be a viable solution if the scenario identifier can be encoded in the interval header in such a way that a non-scenario-aware decoder can still decode scenarioaware video streams. A better and more practical solution is to maintain the scenario information in a separate file or a separate scenario stream. The file or scenario stream can then be sent before or along with the video stream, respectively. The scenario stream solution is particularly interesting because it fits nicely within the container structure that is often used in multimedia streams, for example, the MP4 container format [ISO/IEC 2004].

Note that the amount of scenario information that needs to be sent from the content provider to the client is very limited-in theory, no more than $\left\lceil\log _{2} N_{s}\right\rceil$ bits per interval with $N_{s}$ number of scenarios. In practice, this may require an additional byte, or in some implementations (e.g., in case the scenario information can be embedded in the already existing interval header format), communicating scenario information may not require sending any additional bytes at all. In case additional bytes need to be sent, the scenario information can be highly compressed by exploiting the notion of locality. A video stream typically remains in the same scenario for a given amount of time, that is, the scenario IDs do not change frequently for subsequent intervals of a video stream. We refer to this phenomenon as scenario locality. As such, the scenario IDs that need to be sent from content provider to client can be compressed by capturing the scenario ID of a sequence of subsequent intervals belonging to the same scenario only once.

\section{SCENARIO-DRIVEN DECODING AT THE CLIENT SIDE}

The client can exploit scenarios annotated to the video streams for driving energyefficient decoding. Before actually decoding video streams on the client side, we first need to profile the client by building the scenario adaptation table, as shown in Figure 2. Note that this needs to be done only once per client. The scenario adaptation table summarizes the required cycle count per scenario. Optimal frequencies and voltage levels are then determined at runtime in such a way that an interval belonging to the scenario still meets its deadline, while minimizing the decoder's energy consumption. Once the scenario adaptation table is filled in, that is, the client is calibrated, video streams can then be sent to the client, and the client will adjust the frequency and voltage levels per interval, according to the scenario the interval belongs to. The overall 


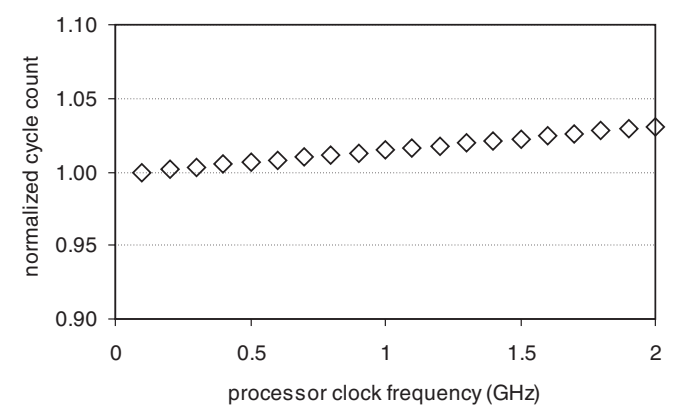

Fig. 3. The impact of processor frequency scaling on cycle count while keeping the memory access latency constant for the H.264/AVC video decoder.

goal is to reduce the energy consumption while meeting the deadlines and preserving the quality of service (QoS).

We now discuss how the client is to be profiled for filling the scenario adaptation table. We subsequently detail the scenario-driven decoding itself and the important additional feature that scenario-based stream annotation offers, namely, being able to predict what the required resources will be for decoding the given video stream of interest.

\subsection{Client Profiling}

In order to enable efficient client profiling, the content provider needs to determine a scenario representative for each scenario (see Figure 2). The scenario representative can be determined as part of the cluster analysis that the content provider needs to do for identifying the video stream scenarios. We select the interval that is closest to the cluster's centroid as the scenario representative. As such, the scenario representatives are intervals taken from various video streams.

The content provider sends these scenario representatives to the client for client profiling. The client then decodes these scenario representatives and monitors how long it takes to decode all of the scenario representatives. Monitoring the required perscenario cycle count can be done using user-accessible hardware performance counters that are available in all modern microprocessors. Once the decode cycle count $C_{\text {decode }}(F)$ at frequency $F$ is known for a given scenario, the scenario operating frequency $F_{\text {decode }}$ is computed as

$$
F_{\text {decode }}=\theta \times \frac{C_{\text {decode }}(F)}{T_{\text {deadline }}},
$$

with $T_{\text {deadline }}$ being the time between two deadlines (in our case, $30 \mathrm{~ms}$ for frame-level scenarios and $480 \mathrm{~ms}$ for GOP-level scenarios). The assumption being made in this formula is that the cycle count remains constant when scaling the clock frequency. This may not be true in general, because the memory access latency remains constant while scaling the processor clock frequency. However, for the video application that we target in this article, this seems to be a very good approximation. This is illustrated in Figure 3, which shows the normalized cycle count as a function of the processor clock frequency, while keeping the memory access latency fixed to 80ns; this graph is computed for the mobile video stream, but we obtained similar results for the other video streams. We observe that the cycle count remains almost constant; the accumulated deviation over the entire $100 \mathrm{MHz}-2 \mathrm{GHz}$ range is less than $3 \%$. This suggests that the video decoder is compute-bound and not memory-bound. 
The $\theta$ parameter in Equation (1) for computing the scenario operating frequency adds an additional scaling parameter; the reason for this scaling parameter is twofold. First, the cycle count is slightly increasing when scaling the clock frequency, as previously mentioned. Second, the scenario representative may not be the interval that requires the maximum cycle count for meeting the deadline among all the intervals for the given scenario. However, given the fact that the variability within a scenario is rather small, this scaling parameter is fairly close to 1 in practice. The $\theta$ threshold is set to 1.15 and 1.1 for the frame-level and GOP-level interval granularity, respectively.

The end result of the client profiling process is a filled in scenario adaptation table, which summarizes the required cycle count per scenario. At runtime, the scenario operating voltage level is then determined based on the scenario operating frequency, which is dependent on the chip technology in which the processor is built. In other words, client profiling translates platform-independent scenario information into platformdependent hardware decode parameters.

\subsection{Scenario-Driven Decoding}

3.2.1. Scenario-Driven Frequency and Voltage Scaling. Scenario-driven video decoding is fairly straightforward once the scenario adaptation table is available. Upon a new interval, the scenario info is read from the interval header or the scenario stream, and the scenario operating frequency and voltage level are determined from the scenario adaptation table and are established in the processor. The process of changing frequency and voltage level at runtime is a well-known mechanism called dynamic voltage and frequency scaling (DVFS).

Frequency and voltage scaling are well-understood mechanisms that are very powerful in reducing energy consumption [Brooks et al. 2000]. In a region where clock frequency and voltage are linear to each other, the dynamic power consumption is $\mathrm{cu}-$ bic in the voltage or frequency. As such, reducing the frequency by a factor $R$ results in a $\propto R^{3}$ reduction in power consumption or a $\propto R^{2}$ reduction in energy consumption. Because of this cubic relationship to power and quadratic relationship to energy, DVFS is a very popular technique in energy-efficient design. In fact, several commercial processors employ DVFS, for example, the Intel XScale processor [Intel 2004] and the Transmeta Crusoe processor [Transmeta Corporation 2001].

3.2.2. Scenario-Driven Resource Prediction. An important feature of our scenario-based mechanism (next to enabling energy-efficient decoding) is that scenarios allow for predicting the resources that the decoding platform require for decoding a given video stream. Some of the required resources that can be predicted using our scheme are compute power and energy consumption. Predicting required resources for a given service is an important feature that enables the end user to decide whether or not to acquire the service. For example, if a high-complex high-resolution video stream is demanded, the content provider may warn the end user that the resources on the decoding platform will not be sufficient for guaranteeing a given quality of service (QoS). Or, the end user may be warned that the available battery lifetime will be insufficient to fully display the acquired video stream. The end user may then, for example, acquire the same video stream in a lower resolution or quality, or the content provider may do that automatically.

Predicting the required compute and energy resources can be done through a handshake mechanism between the client and the content provider. The client communicates the contents of the scenario adaptation table to the content provider once the client profiling is finished. The content provider then determines what the operating frequency and voltage should be at the client side for decoding a given video stream of interest. As such, the content provider can determine how many intervals the client 


Table I. Video Streams and Abbreviations along with
Their JVT [ISO/IEC 2001] Classification
\begin{tabular}{|l|c|c|}
\hline Video stream & Abbr & JVT class \\
\hline akiyo & $\mathrm{ak}$ & $\mathrm{A}$ \\
coast guard & $\mathrm{cg}$ & $\mathrm{B}$ \\
container & con & $\mathrm{A}$ \\
foreman & for & $\mathrm{B}$ \\
hall monitor & hall & $\mathrm{A}$ \\
head w/ glasses & hd & $\mathrm{A}$ \\
mobile & mob & $\mathrm{C}$ \\
mother daughter & md & $\mathrm{A}$ \\
news & news & $\mathrm{B}$ \\
silent & sil & $\mathrm{B}$ \\
stefan & ste & $\mathrm{C}$ \\
table & tab & $\mathrm{C}$ \\
\hline
\end{tabular}

will be unable to decode within the deadlines. (This could happen in case in which the required operating frequency is higher than the maximum clock frequency of the client's processor). Likewise, the content provider can determine what the required energy will be on the client side for decoding the given video stream. A negotiation between the content provider and client can then determine what service quality to deploy to the client.

\section{EXPERIMENTAL SETUP}

Our experiments are done using the H.264 Advanced Video Coding (AVC) codec [Ostermann et al. 2004]. AVC is the new generation compression algorithm for consumer digital video. AVC is the result of work started by ITU and MPEG and was completed by the Joint Video Team (JVT). The algorithm is published as H.264 by the ITU, while ISO/IEC published it as MPEG-4 Part 10. In our measurements, we use version JM6.1 of the reference software of the JVT/AVC codec [Sühring].

In our evaluations, we use twelve video sequences, listed in Table I. These video sequences are widely used in research and can be classified into three classes, according to JVT [ISO/IEC 2001], based on the amount of movement and spatial detail. These chosen video sequences display a wide variety in the amount of spatial detail and movement. A class A video sequence exhibits "low spatial detail and low amount of movement;" class B exhibits "medium spatial detail and low amount of movement or vice versa;" and class C exhibits "high spatial detail and medium amount of movement or vice versa." Each video sequence contains 297 frames; this corresponds to approximately ten seconds of video at a decode rate of 30 frames per second. The results presented in this article are obtained for these video streams in CIF resolution $(352 \times 288$ pixels per frame $)$ and QCIF resolution $(176 \times 144$ pixels per frame). Further, we consider content-adaptive variable-length coding (CAVLC) and a GOP structure containing one I frame followed by $15 \mathrm{P}$ frames.

The performance results presented in this article were obtained using detailed cycle-level processor simulations using the SimpleScalar/Alpha v3.0 tool set [Burger and Austin 1997]. Microprocessor energy consumption is estimated using Wattch v1.02 [Brooks et al. 2000], assuming a 0.18um technology, $2 \mathrm{~V}$ supply voltage, $0.5 \mathrm{~V}$ threshold voltage, and aggressive clock gating, which shuts off unused parts of the microarchitecture while accounting for $10 \%$ leakage energy consumption. These simulation tools were extended to model frequency scaling as well as voltage scaling. When applying frequency scaling, we vary the frequency range from $200 \mathrm{MHz}$ up to $2.7 \mathrm{GHz}$ in $10 \mathrm{MHz}$ and $100 \mathrm{MHz}$ steps and evaluate its impact. When applying both frequency and voltage scaling, we vary voltage with frequency based on $f \propto \frac{\left(V-V_{t h}\right)^{\alpha}}{V}[$ Hughes et al. 
Table II. Four-Wide (Baseline) and Two-Wide Superscalar Processor Models

\begin{tabular}{|c|c|}
\hline \multicolumn{2}{|c|}{ Four-wide (baseline) superscalar processor } \\
\hline Window ROB/LSQ & $32 / 16$ \\
\hline Cache hierarchy & 64KB L1 I/D-caches, 1MB unified L2 \\
\hline Latencies (L1/L2/MEM) & L1: 2 cycles; L2: 20 cycles; MEM: 80ns \\
\hline Branch predictor & Hybrid $4 \mathrm{~K}$ tables, 3 cycle front-end pipeline \\
\hline Processor width & 4-wide \\
\hline Functional units & 4 integer ALUs, 2 memory ports \\
\hline \multicolumn{2}{|c|}{ Two-wide superscalar processor } \\
\hline Window ROB/LSQ & $16 / 8$ \\
\hline Cache hierarchy & 32KB L1 I/D-caches, $128 \mathrm{~KB}$ unified L2 \\
\hline Latencies (L1/L2/MEM) & L1: 2 cycles; L2: 16 cycles; MEM: 80ns \\
\hline Branch predictor & Hybrid 1K tables, 3 cycle front-end pipeline \\
\hline Processor width & 2-wide \\
\hline Functional units & 2 integer ALUs, 1 memory port \\
\hline
\end{tabular}

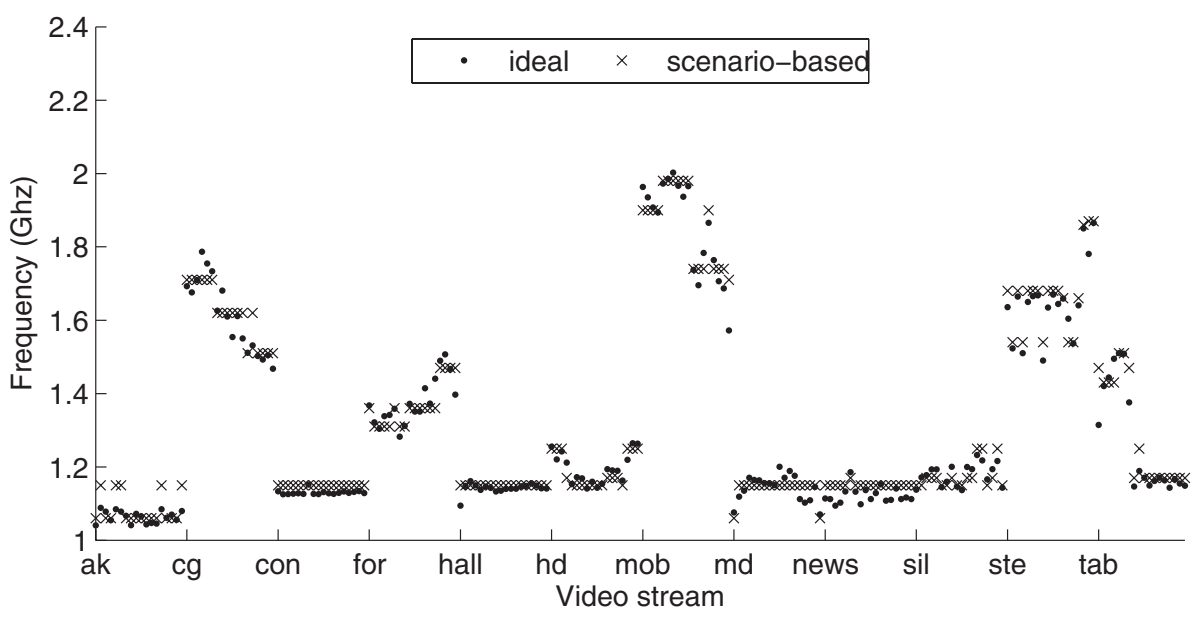

Fig. 4. Tracking decode complexity using 12 GOP-level scenarios.

2001]. We also model the time cost for changing the processor operating frequency: 70 microseconds, according to Burd et al. [2000]. This inter-scenario switching cost (70 microseconds) is much shorter than the inter-frame deadline (30 milliseconds). The baseline processor model used in this article is a contemporary 4-wide superscalar microarchitecture, that is, up to four instructions can be issued per cycle; see Table II. In our experimental evaluation, we also considered a very different processor configuration, namely a 2-wide processor configuration (see Table II) with a reduced number of functional units, reduced cache sizes, and reduced branch-predictor table sizes.

\section{EVALUATION}

\subsection{Scenarios Correlate with Decode Complexity}

Before evaluating the energy-efficiency of scenario-based video decoding and the accuracy of resource prediction, we first evaluate how well the scenarios track decode complexity. For this purpose, we set the scenario-operating-frequency scaling factor $\theta$ to 1.0, that is, deadlines will be missed frequently, and plot the scenario operating frequency versus the minimal ideal processor frequency that still meets the deadline, which is a measure for the decode complexity. Figures 4 and 5 show these plots for GOP-level scenarios (for all video streams) and frame-level scenarios (for the table 




Fig. 5. Tracking decode complexity using 32 frame-level scenarios for the table video stream.

video stream), respectively. The frame-level scenario graph in Figure 5 corresponds to the right-most GOP-level curve (tab) in Figure 4. These graphs clearly show that the scenarios are capable of tracking the decode complexity very well. For example, for the table video stream in Figure 5, the first part of the video stream seems to be much more complex than the second part; the frame-level scenarios clearly track this change in decode complexity fairly well. The frame-level scenarios also discriminate I frames from $\mathrm{P}$ frames; the points around $1.7+\mathrm{GHz}$ are all I frames. Figures 4 and 5 also show that the variability in decode complexity is less for GOP-sized intervals compared to frame-level intervals. The reason is that the decode complexity of the individual frames gets averaged out in case of GOP-sized intervals, that is, the high decode complexity for particular frames may be offset by the low decode complexity for other frames in the same GOP. Note, however, that GOP-sized intervals require buffering at the decoder side to buffer individual frames, which may complicate the client-side decoder.

On average, there are six to seven frame-level scenarios per video stream, assuming 32 scenarios in total; see Table III. The number of frame-level scenarios per video stream varies from three (akiyo, hall monitor, and mother daughter) up to 18 (stefan). In case of GOP-level scenarios (assuming 12 scenarios in total), there are one to five scenarios per video stream; see Table IV.

The transition rate between scenarios across consecutive frames, that is, the probability that consecutive frames belong to a different scenario, equals $26 \%$ on average-from $13 \%$ (hall monitor) up to $44 \%$ (stefan). For GOP-level scenarios, some video streams stay in the same scenario for the entire duration of the video stream, while others, such as stefan, show a transtition rate of up to $33 \%$. 
Table III. Number of Frames (out of 297) per Video Stream (Column) Belonging to a Scenario (Row)

\begin{tabular}{|r||r|r|r|r|r|r|r|r|r|r|r|r|}
\hline & ak & \multicolumn{1}{c|}{ cg } & \multicolumn{1}{c|}{ con } & \multicolumn{1}{c|}{ for } & \multicolumn{1}{c|}{ hall } & hd & mob & md & news & sil & ste & tab \\
\hline$\# \# 1$ & 0 & 0 & 0 & 1 & 0 & 18 & 0 & 0 & 0 & 19 & 0 & 0 \\
\hline$\# 2$ & 0 & 0 & 19 & 0 & 0 & 0 & 0 & 0 & 0 & 0 & 0 & 17 \\
\hline$\# 3$ & 0 & 0 & 0 & 0 & 0 & 0 & 18 & 0 & 0 & 0 & 2 & 0 \\
\hline$\# 4$ & 0 & 3 & 0 & 0 & 0 & 0 & 12 & 0 & 0 & 0 & 145 & 0 \\
\hline$\# 5$ & 0 & 0 & 0 & 19 & 0 & 0 & 0 & 0 & 0 & 0 & 0 & 0 \\
\hline$\# 6$ & 0 & 0 & 0 & 20 & 0 & 0 & 0 & 0 & 0 & 0 & 0 & 0 \\
\hline$\# 7$ & 0 & 40 & 0 & 0 & 0 & 0 & 0 & 0 & 0 & 0 & 0 & 0 \\
\hline$\# 8$ & 0 & 145 & 0 & 8 & 0 & 0 & 0 & 0 & 0 & 0 & 2 & 55 \\
\hline$\# 9$ & 0 & 0 & 0 & 0 & 0 & 0 & 10 & 0 & 0 & 0 & 0 & 0 \\
\hline$\# 10$ & 0 & 0 & 0 & 0 & 0 & 0 & 9 & 0 & 0 & 0 & 0 & 0 \\
\hline$\# 11$ & 0 & 0 & 1 & 121 & 1 & 0 & 0 & 0 & 0 & 0 & 0 & 7 \\
\hline$\# 12$ & 0 & 8 & 0 & 60 & 0 & 0 & 1 & 0 & 0 & 0 & 15 & 14 \\
\hline$\# 13$ & 0 & 0 & 0 & 0 & 0 & 0 & 0 & 0 & 0 & 0 & 4 & 0 \\
\hline$\# 14$ & 0 & 2 & 0 & 0 & 0 & 0 & 0 & 0 & 0 & 0 & 0 & 0 \\
\hline$\# 15$ & 0 & 0 & 0 & 0 & 0 & 0 & 0 & 0 & 0 & 0 & 18 & 0 \\
\hline$\# 16$ & 0 & 17 & 0 & 0 & 0 & 0 & 80 & 0 & 0 & 0 & 5 & 0 \\
\hline$\# 17$ & 0 & 0 & 0 & 31 & 0 & 0 & 0 & 0 & 0 & 0 & 0 & 20 \\
\hline$\# 18$ & 0 & 0 & 0 & 0 & 0 & 0 & 5 & 0 & 0 & 0 & 38 & 0 \\
\hline$\# 19$ & 0 & 0 & 0 & 0 & 0 & 29 & 2 & 0 & 2 & 5 & 3 & 0 \\
\hline$\# 20$ & 76 & 0 & 253 & 19 & 277 & 249 & 0 & 248 & 228 & 261 & 1 & 179 \\
\hline$\# 21$ & 0 & 0 & 0 & 0 & 0 & 0 & 156 & 0 & 0 & 0 & 10 & 0 \\
\hline$\# 22$ & 0 & 63 & 0 & 0 & 0 & 0 & 4 & 0 & 0 & 0 & 5 & 0 \\
\hline$\# 23$ & 0 & 0 & 0 & 0 & 0 & 0 & 0 & 0 & 0 & 0 & 11 & 0 \\
\hline$\# 24$ & 0 & 0 & 0 & 0 & 0 & 0 & 0 & 0 & 0 & 0 & 7 & 2 \\
\hline$\# 25$ & 0 & 0 & 0 & 12 & 0 & 0 & 0 & 0 & 19 & 0 & 0 & 1 \\
\hline$\# 26$ & 19 & 0 & 0 & 2 & 19 & 0 & 0 & 19 & 0 & 0 & 0 & 0 \\
\hline$\# 27$ & 202 & 0 & 24 & 0 & 0 & 0 & 0 & 30 & 48 & 12 & 0 & 2 \\
\hline$\# 28$ & 0 & 0 & 0 & 0 & 0 & 0 & 0 & 0 & 0 & 0 & 7 & 0 \\
\hline$\# 29$ & 0 & 0 & 0 & 0 & 0 & 0 & 0 & 0 & 0 & 0 & 12 & 0 \\
\hline$\# 30$ & 0 & 0 & 0 & 0 & 0 & 0 & 0 & 0 & 0 & 0 & 11 & 0 \\
\hline$\# 31$ & 0 & 0 & 0 & 0 & 0 & 0 & 0 & 0 & 0 & 0 & 1 & 0 \\
\hline$\# 32$ & 0 & 19 & 0 & 4 & 0 & 1 & 0 & 0 & 0 & 0 & 0 & 0 \\
\hline & & & & & & & & & & & & 0 \\
\hline
\end{tabular}

Table IV. Number of GOPs (out of 18) per Video Stream (Column) Belonging to a Scenario (Row)

\begin{tabular}{|r||r|r|r|r|r|r|r|r|r|r|r|r|}
\hline & ak & \multicolumn{1}{|c|}{ cg } & \multicolumn{1}{c|}{ con } & \multicolumn{1}{c|}{ for } & hall & hd & mob & md & news & sil & ste & tab \\
\hline \hline$\# 1$ & 13 & 0 & 0 & 0 & 0 & 0 & 0 & 2 & 0 & 0 & 0 & 0 \\
\hline$\# 2$ & 5 & 0 & 18 & 0 & 18 & 12 & 0 & 16 & 18 & 15 & 0 & 10 \\
\hline$\# 3$ & 0 & 0 & 0 & 0 & 0 & 0 & 0 & 0 & 0 & 0 & 2 & 0 \\
\hline$\# 4$ & 0 & 0 & 0 & 0 & 0 & 0 & 11 & 0 & 0 & 0 & 0 & 0 \\
\hline$\# 5$ & 0 & 0 & 0 & 0 & 0 & 0 & 0 & 0 & 0 & 0 & 0 & 3 \\
\hline$\# 6$ & 0 & 12 & 0 & 0 & 0 & 0 & 0 & 0 & 0 & 0 & 0 & 2 \\
\hline$\# 7$ & 0 & 0 & 0 & 0 & 0 & 6 & 0 & 0 & 0 & 3 & 0 & 1 \\
\hline$\# 8$ & 0 & 0 & 0 & 18 & 0 & 0 & 0 & 0 & 0 & 0 & 0 & 2 \\
\hline$\# 9$ & 0 & 6 & 0 & 0 & 0 & 0 & 1 & 0 & 0 & 0 & 0 & 0 \\
\hline$\# 10$ & 0 & 0 & 0 & 0 & 0 & 0 & 6 & 0 & 0 & 0 & 2 & 0 \\
\hline$\# 11$ & 0 & 0 & 0 & 0 & 0 & 0 & 0 & 0 & 0 & 0 & 13 & 0 \\
\hline$\# 12$ & 0 & 0 & 0 & 0 & 0 & 0 & 0 & 0 & 0 & 0 & 1 & 0 \\
\hline
\end{tabular}

\subsection{Scenario-Driven Energy-Efficient Video Decoding}

We now evaluate scenario-based video decoding in terms of energy reduction and missed deadlines. In all of these experiments, we assume a leave-one-out methodology. This means that when evaluating the efficacy of scenario-based video decoding for a given video stream, we leave that video stream out of the content provider's database for building the scenarios. This leave-one-out evaluation method reflects what is to be 

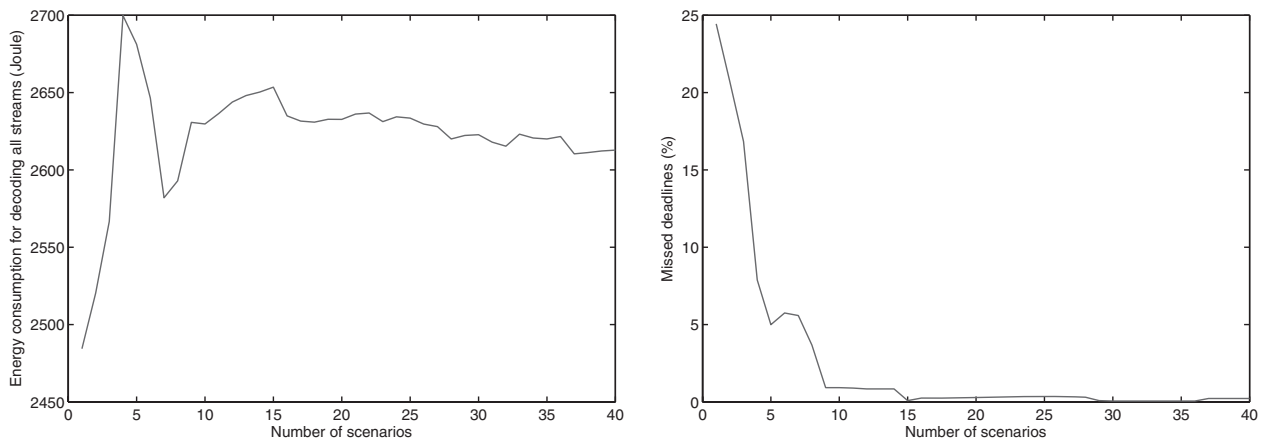

Fig. 6. Energy consumption (on the left) and fraction at missed deadlines (on the right) as a function of the number of frame-level scenarios.



Fig. 7. Normalized energy reduction through frame-level scenario-based video decoding; there are 32 scenarios, in total.

expected in practice whenever a new video stream is added to the content provider's database.

5.2.1. Frame-Level Scenarios. We first focus on frame-level scenarios. Figure 6 shows the energy consumption (on the left) and the fraction of missed deadlines (on the right) as a function of the number of scenarios. We observe that the energy consumption fluctuates with the number of scenarios; however, the variation is fairly small (no more than a few percent). The fraction of missed deadlines, on the other hand, is fairly sensitive to the number of scenarios. Less than 15 scenarios result in a relatively high number of missed deadlines. By consequence, the energy reduction obtained with a limited number of scenarios is mainly due to tolerating missed deadlines. This is not acceptable, of course. Around 28 to 37 scenarios of all of the video streams have no missed deadlines, except for foreman, for which $0.7 \%$ of all its frames miss their deadline.

Figure 7 shows normalized energy consumption for each of the video streams, while assuming 32 frame-level scenarios. The energy consumption is normalized to the energy consumption while decoding all the video streams at a single fixed-clock frequency that guarantees that all frames in all video streams are decoded within the deadlines. In other words, the minimum clock frequency is determined by the most computation-demanding frame in all of the video streams. This is the 'reference' bar 

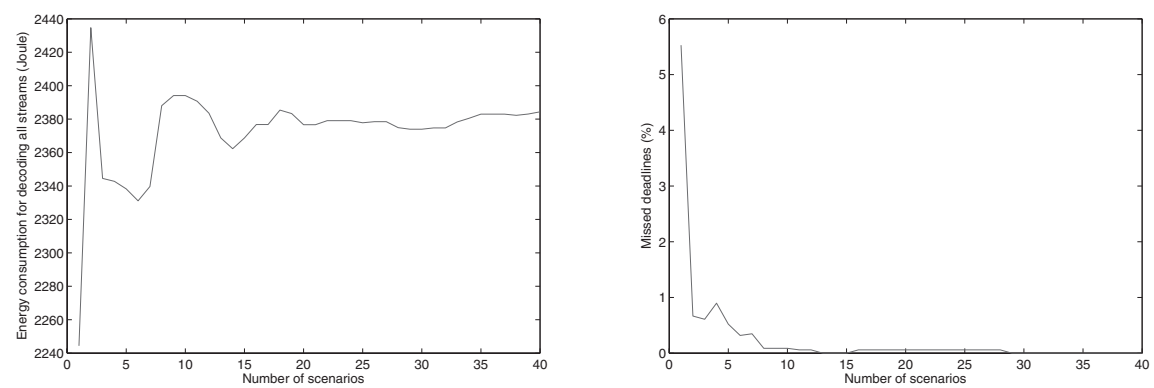

Fig. 8. Energy consumption (on the left) and fraction at missed deadlines (on the right) as a function of the number of GOP-level scenarios.

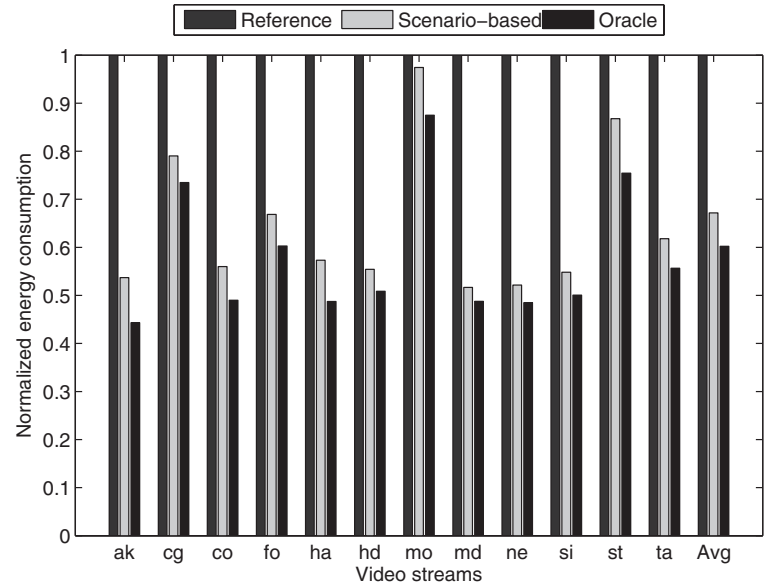

Fig. 9. Normalized energy reduction through GOP-level scenario-based video decoding; there are 12 scenarios in total.

in Figure 7. The 'oracle' bar shows the normalized energy consumption while decoding all the video stream intervals at the minimum clock frequency per interval; this cannot be achieved in practice unless an oracle mechanism is assumed. We observe that the maximum achievable energy reduction is high (more than 60\%) for most of the video streams; the most computation-demanding video stream is mobile, for which the oracle video decoding mechanism cannot reduce the energy reduction consumption by more than 33\%. Scenario-based video decoding is capable of achieving most of the potential energy reduction. On average, frame-level scenario-based decoding reduces energy consumption by as much as $44 \%$, whereas the oracle mechanism achieves an average reduction of $52 \%$.

5.2.2. GOP-Level Scenarios. Figure 8 shows energy consumption and fraction of missed deadlines as a function of the number of GOP-level scenarios. Once beyond eight GOPlevel scenarios, the number of missed deadlines becomes very small, and energy consumption seems to stabilize. The fact that fewer GOP-level scenarios are required than frame-level scenarios can be explained by the fact that there are more extreme behaviors at the frame level; grouping multiple frames at the GOP level has a smoothing or averaging effect on the extreme frame-level behaviors.

Figure 9 shows the energy reduction per video stream, relative to the case in which all GOPs are decoded within their deadlines. This graph assumes 12 GOP-level scenarios. 


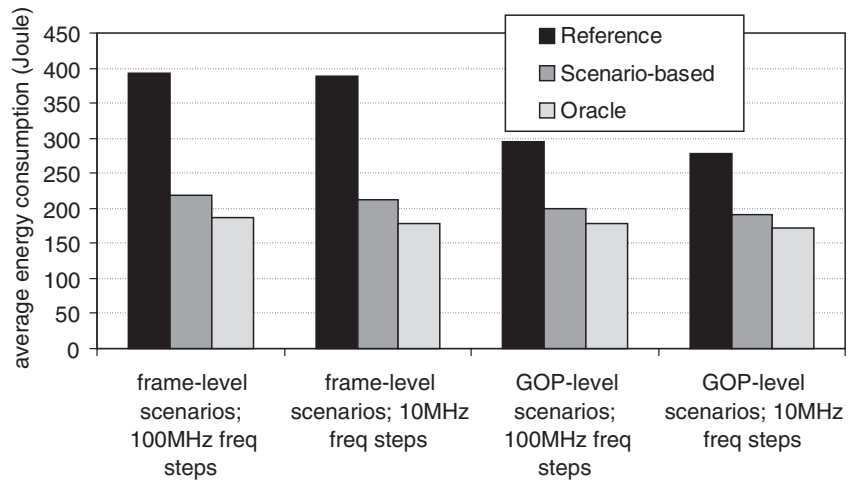

Fig. 10. Average energy consumption per video stream for frame-level versus GOP-level scenarios and $10 \mathrm{MHz}$ versus $100 \mathrm{MHz}$ DVFS frequency steps; these results assume CIF resolution video streams.

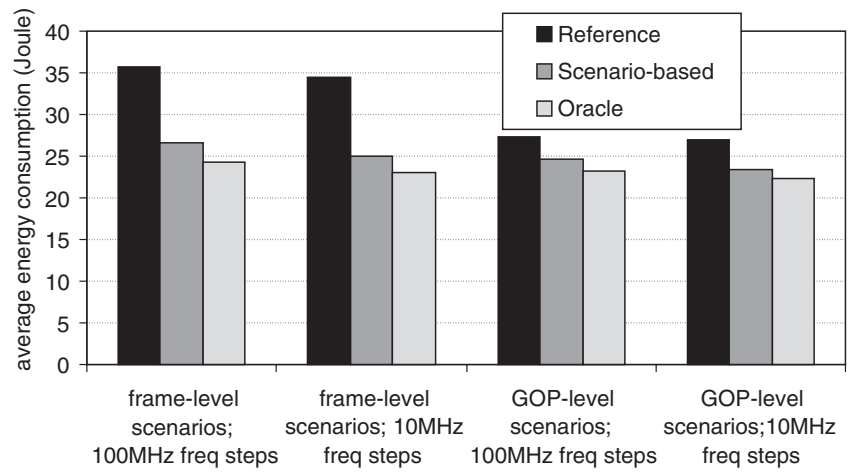

Fig. 11. Average energy consumption per video stream for frame-level versus GOP-level scenarios and $10 \mathrm{MHz}$ versus $100 \mathrm{MHz}$ DVFS frequency steps; these results assume QCIF resolution video streams.

There are no missed deadlines in this setup for all of the video streams, except for the foreman video stream, for which $0.7 \%$ of all frames miss their deadline. Note that we count the number of missed deadlines as the number of frames that are not decoded by the end of the GOP's deadline. This assumes a buffering mechanism in the video decoder. The potential energy reduction that can be achieved for GOP-level scenarios is $40 \%$. Scenario-based video decoding with GOP-level scenarios achieves a 33\% reduction in overall energy consumption.

Comparing frame-level scenarios versus GOP-level scenarios, we observe that the relative energy consumption reduction is larger for frame-level scenarios than for GOPlevel scenarios (for the same number of missed deadlines). The reason is that, as mentioned before, there is more variability in decode complexity at the frame level than at the GOP level. This requires that more scenarios are to be identified at the frame level, which results in more energy-saving opportunities.

5.2.3. Impact of Video Resolution and DVFS Granularity. Figures 10 and 11 show the average energy reduction per video stream for CIF and QCIF resolution video streams, respectively. Both graphs explore the impact of frame-level versus GOP-level scenarios, as well as the impact of $100 \mathrm{MHz}$ versus $10 \mathrm{MHz}$ DVFS frequency steps. As mentioned before, we assume 32 frame-level scenarios and 12 GOP-level scenarios; there are virtually no missed frame deadlines. Note that the 'reference' bars in Figures 10 and 11 do not assume the same clock frequency for frame-level and GOP-level scenarios. 




Fig. 12. Average energy reduction compared to the reference run for 2-wide and 4-wide superscalar processors for CIF and QCIF video resolutions, assuming frame-level scenarios.

GOP-level scenarios assume there is a buffering mechanism in place that buffers up to 16 frames. The energy reduction that is potentially achievable through GOP-level scenarios is therefore lower than that at frame-level scenarios, that is, there is a smoothing effect in which low-complexity frames compensate for high-complexity frames, leading to a lower clock frequency, on average, and thus an overall lower energy consumption.

There are several interesting observations to be made here. First, the energy reduction obtained through scenario-aware video decoding is higher for the higher-resolution CIF video streams than for the QCIF video streams; compare the energy savings in Figure 10 versus Figure 11. For example, for frame-level scenarios, we obtain an average $44 \%$ energy consumption reduction for CIF compared to an average $33 \%$ energy consumption reduction for QCIF. The reason is that higher resolution video streams exhibit more extreme decode complexity behaviors, which results in more energy-saving opportunities. The underlying reason for the more extreme decode complexity is that a high resolution frame contains more macroblocks than a low resolution frame, and each macroblock can potentially be encoded in a different format. Second, although the relative energy saving is higher for frame-level scenarios than for GOP-level scenarios, as explained in the previous section, the absolute energy consumption for GOP-level scenario-aware decoding is slightly lower compared to frame-level scenario-aware decoding. This would suggest that GOP-level scenarios is are less complex, and therefore a better design choice than frame-level scenarios, since there are fewer GOP-level scenarios than frame-level scenarios. Note, however, that GOP-level scenarios assume there is a buffering mechanism in place at the client side to buffer decoded frames. Third, the energy consumption is comparable for the $100 \mathrm{MHz}$ and $10 \mathrm{MHz}$ DVFS frequency steps. We thus conclude that scenario-aware video decoding does not require a fine-grain DVFS mechanism; a coarse-grain DVFS mechanism is as efficient.

5.2.4. Platform-Independence. As previously mentioned, an important assessment of scenario annotation presented in this article is that the scenarios are platformindependent. In other words, the scenarios are identified based on platformindependent decoding complexity by looking at macroblock profiles. As a consequence, scenario information can be exploited on various platforms. Figure 12 illustrates this by showing the percentage of energy reduction compared to the reference run for a 4-wide (our baseline) and a 2-wide superscalar processor. In both cases, the number of missed deadlines is virtually zero. For both processors, we observe a substantial reduction in energy consumption: $44 \%$ for the 4 -wide superscalar processor and $53 \%$ for the 2 -wide 


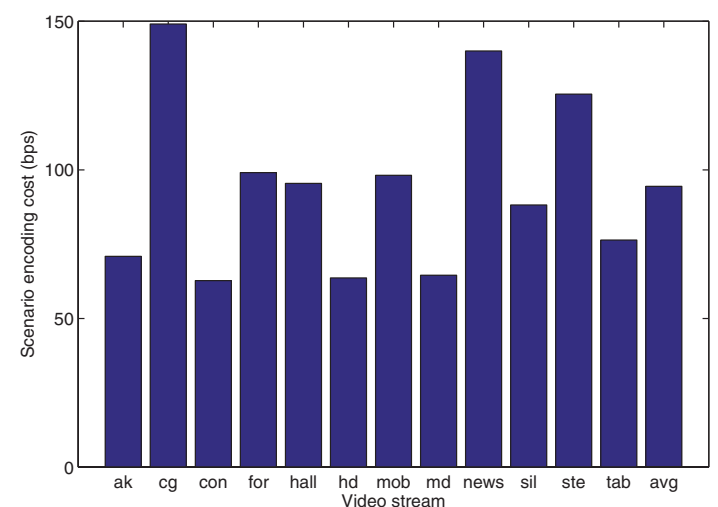

Fig. 13. Cost of scenario annotation in number of bits per second for 32 frame-level scenarios.

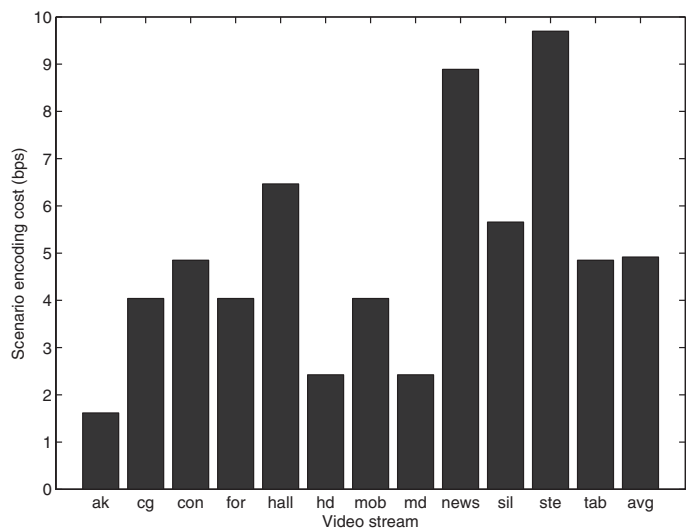

Fig. 14. Cost of scenario annotation in number of bits per second for 12 GOP-level scenarios.

superscalar processor, for CIF video resolution. This result clearly illustrates that the scenarios are platform-independent and can therefore be exploited across platforms.

5.2.5. Scenario-Encoding Cost. Scenario-aware video streams need to be annotated with scenario IDs. Figure 13 quantifies the amount of bits for annotating video streams with frame-level scenarios. Figure 14 presents the same for GOP-level scenarios. These graphs assume CIF resolution, that is, the reported results incur, the largest scenario annotation cost among the experiments conducted in this article. The average cost per video stream, assuming run-length encoding, is less than 100 bits per second for framelevel scenarios. This corresponds to approximately $45 \mathrm{~KB}$ of compressed storage and $66 \mathrm{~KB}$ of uncompressed storage for a one-hour video. Obviously, video streams showing little scenario locality, such as coast guard, require more bits in order to encode scenario information. For GOP-level scenarios, the average encoding cost is about a factor 20 smaller with around 5 bits per second.

\subsection{Resource Prediction}

We now evaluate the proposed scenario-based resource prediction method. We consider three flavors of resource prediction: predicting decode time, predicting quality of service, and predicting energy consumption. We also evaluate resource prediction accuracy 


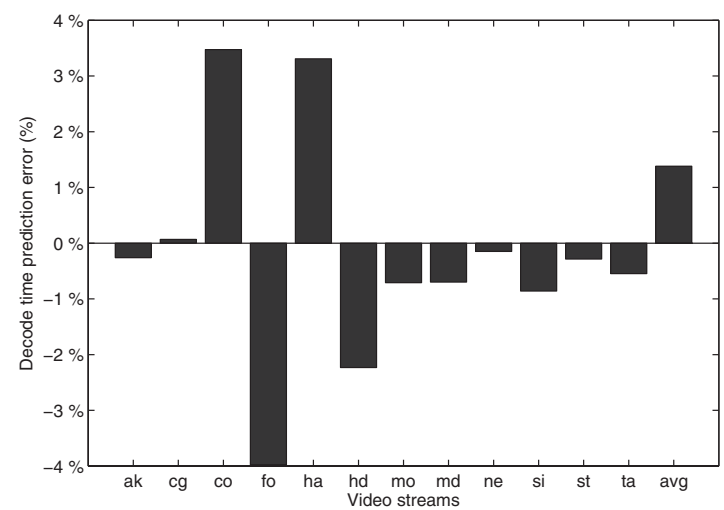

Fig. 15. Per-benchmark decode time prediction errors assuming 32 frame-level scenarios.

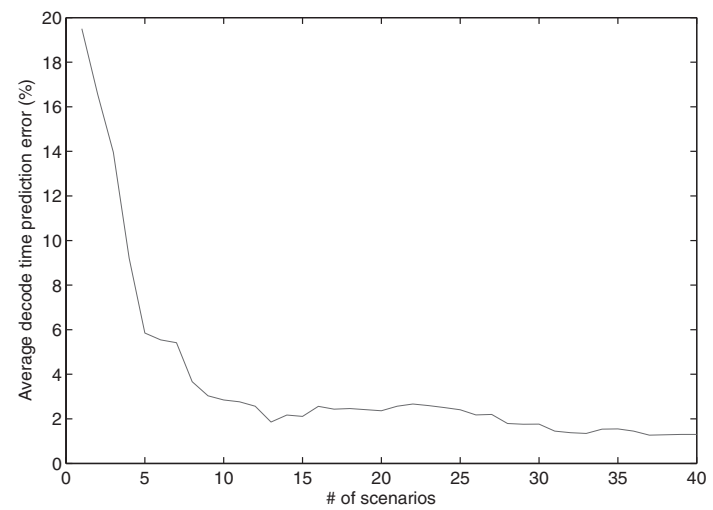

Fig. 16. Average decode time prediction error as a function of the number of scenarios.

across platforms and additionally evaluate frame-level versus GOP-level scenarios for resource prediction.

5.3.1. Predicting Decode Time. We first evaluate the accuracy for predicting the decode time for a given video stream on a given client. When assuming a $2 \mathrm{GHz}$ clock frequency at the client side, ${ }^{1}$ the decode time for decoding the entire video stream can be predicted with an error of at most $4 \%$ and an average error of $1.4 \%$; see Figure 15. This assumes 32 frame-level scenarios. Figure 16 shows the average decode time prediction error as a function of the number of scenarios. We observe that the average prediction error decreases with an increasing number of scenarios; once above ten scenarios, we obtain an average prediction error around $2.5 \%$, which drops to less than $1.5 \%$ once above 30 scenarios.

Figure 17 gives a more detailed view by showing the cumulative distribution function of per-frame decode time prediction errors. A point $(x, y)$ on one of the curves states that a decode time prediction error less than $x \%$ is achieved for $y \%$ of the frames; also, for $100 \%-y \%$ of the frames, the decode time prediction error is larger than $x \%$. This graph once again shows that prediction error decreases with an increasing number of scenarios. Assuming a single scenario, $65 \%$ of all frames show a prediction error larger

${ }^{1}$ We have to set the client clock frequency to $2 \mathrm{GHz}$ in order to meet the $30 \mathrm{~ms}$ frame deadlines using the reference decoder. 




Fig. 17. Predicting individual frame decode times. The percentage frames (on the vertical axis) for which the decode time prediction error is less than a given percentage (on the horizontal axis).

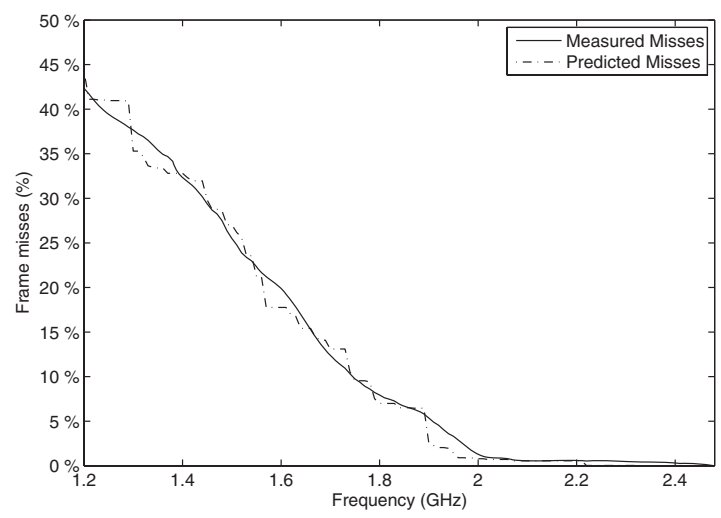

Fig. 18. Predicting the number of missed frame deadlines as a function of the client's clock frequency (in $10 \mathrm{MHz}$ steps) on the horizontal axis.

than $20 \%$. However, once there are more than 20 scenarios, $90 \%$ of all frames have a decode time prediction error of less than $6.3 \%$. Or, only $3.2 \%$ of all frames have a decode time prediction error larger than $10 \%$.

5.3.2. Predicting Quality of Service. Resource prediction can also be used to predict quality of service. In particular, the content provider can predict whether the client platform is computationally powerful enough for decoding a given video stream. In other words, the content provider can make an estimate of the number of frame deadlines that will be missed by the client. Figure 18 quantifies the accuracy for predicting the quality of service. The two curves show the real percentage of missed frame deadlines and the predicted percentage of missed frame deadlines as a function of the client's clock frequency. When running the client's clock frequency at $1.2 \mathrm{GHz}$, approximately $40 \%$ of all frame deadlines will be missed; however, at $2.5 \mathrm{GHz}$, no deadlines will be missed. This graph clearly shows that the estimated number of missed frame deadlines matches the real number of missed frame deadlines very closely over the entire client's clock frequency range. The prediction error is never larger than $4 \%$.

5.3.3. Predicting Energy Consumption. We now evaluate the accuracy of scenario-based video stream analysis for predicting the overall energy consumption. The results are shown in Figure 19 for decoding at a $2 \mathrm{GHz}$ clock frequency. We obtained similar results 


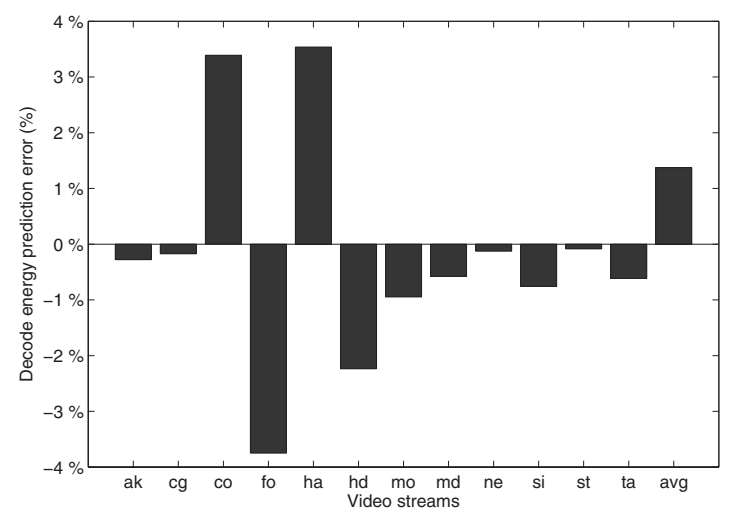

Fig. 19. Energy prediction error for a client decoding at a fixed $2 \mathrm{GHz}$ clock frequency.

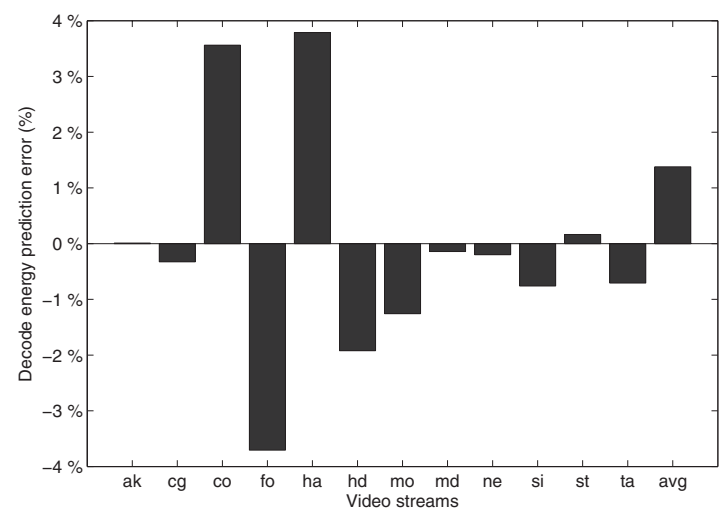

Fig. 20. Energy prediction error for a scenario-aware DVFS client-side video decoder.

over the entire $1.2 \mathrm{GHz}$ to $2.5 \mathrm{GHz}$ range. The average (absolute) prediction error is $1.4 \%$; the maximum prediction error is observed for the container, foreman, and hall monitor video streams $(3.5 \%$ to $3.8 \%)$.

These results were obtained when decoding a video stream at a fixed clock frequency. Figure 20 shows the error when predicting the amount of energy consumed for decoding the given video stream using the DVFS-driven, scenario-aware video decoding method presented in this article. The average prediction error is $1.4 \%$; the maximum prediction error is never larger than $4 \%$. Figure 21 shows the average energy prediction error as a function of the number of frame-level scenarios. This graph is completely in line with the decode time prediction errors from Figure 16: the average prediction error decreases with an increasing number of scenarios and reaches an average error around $2.5 \%$ once beyond ten scenarios and less than $1.5 \%$ once beyond 30 scenarios.

5.3.4. Platform Independence. As previously mentioned, scenarios are platformindependent and can thus be used to predict decode time, quality of service, and energy consumption across platforms. Our results, using two different microprocessor configurations, confirm that scenario-aware resource prediction is accurate on both platforms, that is, the results presented in the previous sections for the four-wide superscalar processor are completely in line with the results observed for the two-wide superscalar processor: for the two-wide superscalar processor, the average decode time and energy consumption prediction error equals $1.7 \%$ and $1.5 \%$, respectively. 


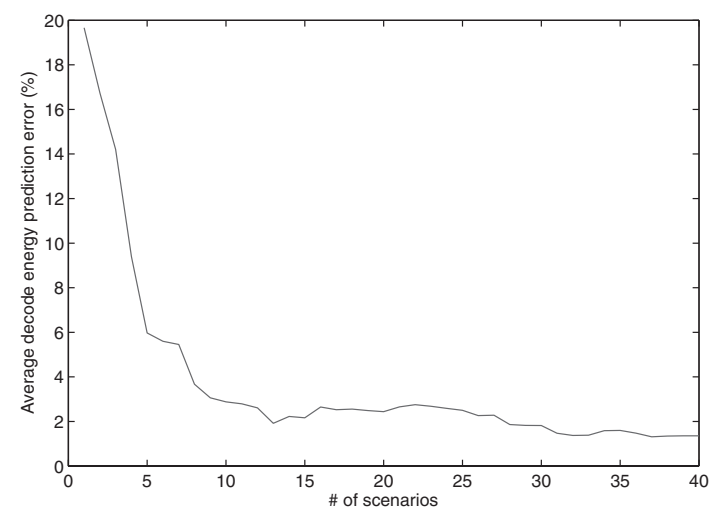

Fig. 21. The average energy prediction error as a function of the number of scenarios.

5.3.5. GOP-Level Scenarios. Resource prediction using GOP-level scenarios instead of frame-level scenarios (as done in the previous sections) is (slightly) less accurate. The average decode time and energy consumption prediction error using GOP-level scenarios equals $2.3 \%$ compared to $1.4 \%$ for frame-level scenarios. The reason is that frame-level scenarios enable a more fine-grained classification of frames in scenarios.

\section{RELATED WORK}

We now discuss related work in characterizing video decoder complexity, resource prediction, and energy-efficient video decoding.

\subsection{Video Decoder Complexity and Resource Prediction}

The following two papers that we will discuss in detail deal with quantifying video decoding complexity.

Horowitz et al. [2003] present a detailed platform-independent characterization of the H.264/AVC video decoder, which is the same video decoder as the one considered in this article. The characterization is done in terms of macroblock types and the number of fundamental operations required to perform the key decoder subfunctions. The platform-independent characterization is then linked with experimental results from three hardware platforms. The macroblock profiling that we consider as input to the scenario identification at the content provider side is similar to the platformindependent characterization done by Horowitz et al. However, they do not study nor exploit similarities within and across video streams.

Mattavelli and Brunetton [1998] also characterize video streams in a platformindependent manner by counting the number of inter prediction macroblocks, the number of intra prediction macroblocks, the number of skip macroblocks, etc. For predicting the decoder performance on a particular platform using these platformindependent metrics, they then employ linear regression to learn how to weight the various platform-independent metrics for making accurate performance predictions. This is similar in design to our approach of client profiling. However, there are two major limitations with the work by Mattavelli and Brunetton compared to our approach. First, Mattavelli and Burnetton require a relatively large set of video sequences with associated measurements. This is impractical on resource-constrained embedded devices, as profiling the client may take considerable time. In case of scenario-based resource prediction, on the other hand, client profiling is done very quickly using only a few frames, namely, the scenario representatives. Second, the storage requirements on the content provider side for Mattavelli and Brunetton's technique is very large (at 
least two orders of magnitude larger) compared to scenario-based resource prediction. Mattavelli and Brunetton need to store regression weights for all platform-independent metrics per frame; this would be hundreds of bits per frame in our setup. We only need a few bits for encoding the scenario ID per frame. Note that the study presented by Mattavelli and Brunetton is limited to resource prediction of decode time only. Our work is much broader in scope: we provide a unified scenario-based framework for both resource prediction and energy-efficient media decoding.

Gheorghita et al. [2005] use scenarios for worst-case execution times (WCET) estimation. WCET estimation is a different problem than resource prediction. WCET estimation needs formally proved execution time bounds; resource prediction, on the other hand, strives at making realistic performance, QoS, and energy estimates.

\subsection{Energy-Efficient Video Decoding}

We make a distinction between client-side and content provider-side solutions to energy-efficient video decoding.

6.2.1. Client-Side Solutions. Several proposals to energy-efficient video decoding have been made in recent literature [Acquaviva et al. 2001; Choi et al. 2002; Gheorghita et al. 2005; Hughes et al. 2001; Lu et al. 2002; Mohapatra et al. 2003; Shin et al. 2001; Yuan and Nahrstedt 2004]. We now discuss this prior work in more detail, particularly focusing on the prior work most closely related to our work. For each of these, the major differences between prior work and our approach are detailed.

Hughes et al. [2001] present a client-side energy-reduction approach that consists of two phases. In the first phase, the profiling phase, an optimal hardware configuration is determined for each frame type. The end result of the profiling step is a table with an entry for each possible hardware configuration stating the maximum budget of instructions for processing a given frame type without missing the deadline. In the second phase, the adaptation phase, the number of instructions of the next frame is predicted, and the hardware configuration is installed for processing the next frame that will minimize energy consumption without missing the deadline. A key point of this approach is how to predict the number of instructions for the next frame. Hughes et al. evaluated a number of instruction count predictors and incorporated a leeway and hysteresis mechanism in their predictor to trade off the fraction of under-predictions and keep track of the variability in instruction count due to changes in the input (e.g., due to scene changes). A similar approach based on predicting the computational workload for the next incoming frame was also proposed by Choi et al. [2002]. Another approach to client-side energy-efficient decoding is by using control-theoretic feedbackcontrol mechanisms, as proposed by Lu et al. [2002]. These approaches are reactive, that is, when the input sequence changes complexity, the mechanism needs to learn how to deal with that. This may result in missed deadlines. Our proposal is radically different from these proposals. Our approach is a proactive scheme, that is, the scenario IDs encode the decoding complexity in the video stream so that the decoder can respond in a timely fashion to decoding complexity changes.

Gheorghita et al. [2005] propose a proactive, scenario-based DVFS approach that is based on a static analysis of the video decoder. They first identify scenarios based on how the input affects the application execution time. For each of the scenarios, they identify where in the source code a scenario starts and ends, and finally, they embed scenario information into the application binary. The inherent limitation of this approach is that it relies on a static analysis of the application and requires recompiling the decoder. In order for this approach to work well, extensive profiling is required in order to identify scenarios across various inputs. And this needs to be done on all the client platforms of interest. This may be infeasible in practice. The results reported 
by Gheorghita et al. only identify two scenarios in a video decoder application: one scenario for decoding I frames and one scenario for decoding $\mathrm{P}$ frames. In other words, this approach seems to be unable to distinguish a video stream segment that shows a hectic action scenery and requires substantially more computational power at decode time than a quiet scenery, although both sceneries may be encoded using the same GOP structure. Our approach, on the other hand, which is also a proactive mechanism, can track such scenery changes effectively by assigning different scenarios to sceneries with different complexities.

All of these energy-efficient video decoding approaches rely on the client for analyzing the input stream complexity. This adds an additional computational burden to the resource-constrained client, which may be infeasible in practice. Offline and content provider assisted scenario annotation alleviates this issue, as discussed next.

6.2.2. Offline and Content Provider Assisted Solutions. Huang et al. [2005] propose an offline bitstream analysis methodology for energy-efficient video decoding. They assume the following setup. The portable device on which the video stream needs to be displayed is assumed to be connected to a desktop computer. It is assumed that an application program runs on this desktop that is specialized for the portable device. Before sending the video stream from the desktop computer to the portable device, the application program analyzes the bitstream and inserts metadata information into the video stream. The metadata inserted into the video stream is specific for the portable device and the video decoder running on the portable device. The meta information encodes frequency and voltage level information for driving the DVFS-aware video decoder and is obtained by predicting the device's processor cycle requirements at the macroblock level. The major limitation of the offline bitstream analysis approach by Huang et al. is that it assumes that the portable device is connected to a desktop computer, which may not be the case in most practical circumstances. In addition, a decoder and device-specific application program to be run on the desktop computer needs to be built for each possible platform of interest. This is both costly, time-consuming, and difficult to maintain in practice. The video stream scenario approach proposed in this article does not suffer from these limitations.

The approach most closely related to our approach is the one proposed by Chung et al. [2002]. The key idea of Chung et al. is to annotate the decoding cost to each frame in a video stream. The decoding cost for a given frame is defined as the decoding time for the given frame, normalized to the decoding time of the first frame in the video stream. Note that because of the normalization, the decoding cost is a platformindependent metric. The decoding cost characterization is done by the content provider by decoding the video stream and timing the decode time. This approach has three major disadvantages compared to our approach. First, the content provider needs to decode all the video streams in the database for computing the decoding costs for the individual frames. We do not require the video streams to be decoded; we just count macroblock types. Second, the decoding cost information that needs to be sent is a 4-byte floating-point number for each frame. The scenario IDs that we use are much more condensed in storage. And finally, the scheme by Chung et al. assumes that the decoding cost at the content provider side correlates very well with the decoding cost at the client side. This is not always the case because the content provider's platform (both the video decoder as well as the processor and system architecture) can be very different from the client's platform. This may yield widely varying decoding costs across both platforms. This is illustrated in Figure 22, which shows normalized decode time proportional to the decode time of the first frame of the video stream akiyo. The frames are ordered based on this normalized decode time for an 8-wide superscalar processor. This is a processor configuration that is to be expected at the content provider side. 


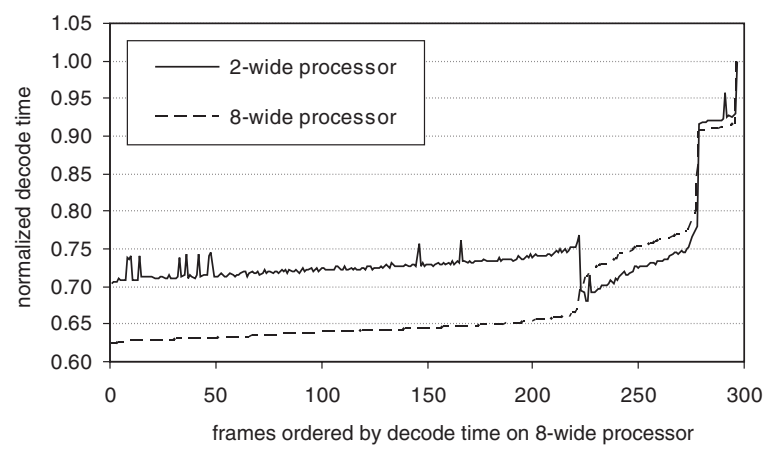

Fig. 22. Decoding cost on a 2-wide and 8-wide superscalar processor normalized to the decoding time of the first frame and sorted by the decoding cost on the 8-wide processor.

We thus observe a monotonically increasing curve for the 8-wide processor. The other curve shows the measured decode time for a 2-wide client processor. We observe that the 2 -wide processor curve shows fairly significant fluctuations, up to $13 \%$ for some frames (see around frame number 220 in Figure 22). A nonstable decoding cost across platforms may result in missed deadlines or lost energy reduction opportunities, in practice.

Next to not suffering from these limitations, our approach has an additional major benefit in that it allows for driving resource prediction, that is, compute power and energy consumption can be predicted based on the scenario information that is annotated to the video streams. None of the previously proposed energy-efficient video decoding approaches (neither the client-side nor the content provider-assisted solutions) have such a capability.

\section{CONCLUSION}

This article proposed an energy-efficient media-decoding methodology based on media stream similarity. The key idea is to identify similarly behaving scenarios, both within and across media streams, in a platform-independent manner. Media streams in a content provider's database are then annotated with scenario information. This article presented two ways of exploiting the scenario information: (i) energy-efficient video decoding at the client side through dynamic voltage and frequency scaling, that is, the client processor's frequency and voltage level are adjusted on a per-scenario basis, and (ii) resource prediction of decode time, energy, and quality of service. Both applications are driven by a single, unifying scenario-based mechanism and framework. To the best of our knowledge, this article is the first to identify and exploit media stream similarity. Our evaluation shows that scenario-based video decoding of the H.264 AVC decoder reduces overall energy consumption by $44 \%$ and $33 \%$ for frame-level and GOPlevel scenarios, respectively, while missing virtually no frame deadlines. An important additional feature of scenario analysis is that it enables accurate compute power and energy resource prediction, with prediction errors less than $4 \%$ and a $1.4 \%$ average prediction error.

\section{REFERENCES}

Acquaviva, A., Benini, L., And Riccó, B. 2001. An adaptive algorithm for low-power streaming multimedia processing. In Proceedings of the Conference on Design, Automation and Test in Europe (DATE). 273-279.

Brooks, D., Bose, P., Schuster, S. E., Jacobson, H., Kudva, P. N., Buyuktosunoglu, A., Wellman, J.-D., Zyuban, V., GuPTA, M., AND Cook, P. W. 2000. Power-aware microarchitecture: Design and modeling challenges for next-generation microprocessors. IEEE Micro. 20, 6, 26-44. 
Brooks, D., Tiwari, V., AND Martonosi, M. 2000. Wattch: A framework for architectural-level power analysis and optimizations. In Proceedings of the 27th Annual International Symposium on Computer Architecture (ISCA'27). 83-94.

Burd, T. D., Pering, T. A., Stratakos, A. J., and Brodersen, R. W. 2000. A dynamic voltage scaled microprocessor system. IEEE J. Solid-State Circuits 35, 11, 1571-1580.

Burger, D. C. And Austin, T. M. 1997. The SimpleScalar tool set. Computer Architecture News. http://www. simplescalar.com.

Choi, K., Dantu, K., Cheng, W.-C., And Pedram, M. 2002. Frame-based dynamic voltage and frequency scaling for a MPEG decoder. In Proceedings of the International Conference on Computer-Aided Design (ICCAD). 732-737.

Chung, E.-Y., Benini, L., ANd De Micheli, G. 2002. Contents provider-assisted dynamic voltage scaling for low energy multimedia applications. In Proceedings of the International Symposium on Low Power Electronic Design (ISLPED). 42-47.

Gheorghita, S. V., Basten, T., and Corporaal, H. 2005. Intra-task scenario-aware voltage scheduling. In Proceedings of the International Conference on Compilers, Architectures and Synthesis for Embedded Systems (CASES). 177-184.

Gheorghita, S. V., Stuijk, S., Basten, T., and Corporaal, H. 2005. Automatic scenario detection for improved WCET estimation. In Proceedings of the 42nd Annual Cnference on Design Automation (DAC). 101-104.

HAMERS, J. AND Eeckhout, L. 2007. Resource prediction for media stream decoding. In Proceedings of the Design, Automation and Test in Europe (DATE). 594-599.

Hamers, J., Eeckhout, L., AND De Bosschere, K. 2007. Exploiting video stream similarity for energy-efficient decoding. In Proceedings of the 13th International Multimedia Modeling Conference Part II. 11-22.

Horowitz, M., Joch, A., Kossentini, F., And Hallapuro, A. 2003. H.264/AVC baseline profile decoder complexity analysis. IEEE Trans. Circuits Syst. Video Technol. 13, 7, 704-716.

HuANG, Y., CHAKRABorty, S., AND WANG, Y. 2005. Using offline bitstream analysis for power-aware video decoding in portable devices. In Proceedings of the 13th Annual ACM International Conference on Multimedia (MM). 299-302.

Hughes, C. J., SRinivasan, J., AND Adve, S. V. 2001. Saving energy with architectural and frequency adaptations for multimedia applications. In Proceedings of the 34th Annual International Symposium on Microarchitecture (MICRO'34). 250-261.

Intel 2004. Intel XScale Core Developer's Manual. Intel. 273473-002.

ISO/IEC. 2001. MPEG-4 video verification model version 18.0. ISO/IEC JTC1/SC29/WG11 N3908.

ISO/IEC. 2004. Information technology—coding of audio-visual objects—part 14: Mp4 file format. ISO/IEC 14496-14:2003.

Johnson, R. A. And Wichern, D. W. 2002. Applied Multivariate Statistical Analysis 5th Ed. Prentice Hall.

Lu, Z., Hein, J., Humphrey, M., Stan, M., Lach, J., and Skadron, K. 2002. Control-theoretic dynamic frequency and voltage scaling for multimedia workloads. In Proceedings of the International Conference on Compilers, Architectures and Synthesis for Embedded Systems (CASES). 156-163.

MattavelLi, M. AND BRunetton, S. 1998. Implementing real-time video decoding on multimedia processors by complexity prediction techniques. IEEE Trans. Consum. Electron. 44, 3, 760-767.

Mohapatra, S., Cornea, R., Dutt, N., Nicolau, A., and VaKatasubramanian, N. 2003. Integrated power management for video streaming to mobile handheld devices. In Proceedings of the 11th Annual ACM International Conference on Multimedia (MM). 582-591.

Ostermann, J., Bormans, J., List, P., Marpe, D., Narroschke, M., Pereira, F., Stockhammer, T., and Wedi, T. 2004. Video coding with H.264/AVC: Tools, performance and complexity. IEEE Circuits Syst. Mag. 4, 1, $7-28$.

SHIN, D., KIM, J., AND LEE, S. 2001. Intra-task voltage scheduling for low-energy, hard real-time applications. IEEE Des. Test Comput. 18, 2, 20-30.

SüHRING, K. H.264/AVC reference software. http://iphome.hhi.de/suehring/tml/download/.

Transmeta Corporation 2001. LongRun Power Management: Dynamic Power Management for Crusoe Processors. Transmeta Corporation.

Yuan, W. ANd Nahrstedt, K. 2004. Practical voltage scaling for mobile multimedia devices. In Proceedings of the 12th Annual ACM International Conference on Multimedia (MM). 924-931.

Received June 2007; revised October 2007; accepted March 2008 